\title{
Perfil de ácidos grasos de diversas especies de pescados consumidos en México
}

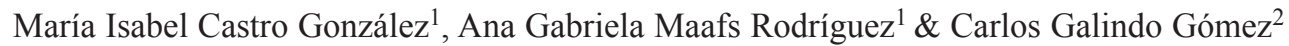 \\ 1. Departamento de Nutrición Animal, Instituto Nacional de Ciencias Médicas y Nutrición Salvador Zubirán, Vasco de \\ Quiroga N¹5, Col. Sección XVI. Del. Tlalpan, C.P. 14000, México, D.F.; isacastro55@yahoo.com.mx, \\ anga022@hotmail.com \\ 2. Dirección de Nutrición, Instituto Nacional de Ciencias Médicas y Nutrición Salvador Zubirán, Vasco de Quiroga \\ N¹5, Col. Sección XVI. Del. Tlalpan, C.P. 14000, México, D.F.; cgg68@hotmail.com
}

Recibido 12-X-2012. C Corregido 20-II-2013. Aceptado 13-III-2013.

\begin{abstract}
Fatty acids in different edible fish species from Mexico. Different biotic and abiotic factors determine the fatty acid (FA) composition of fish tissues and organs. This information is useful for humans due to the fact that fish consumption is associated with health benefits. The aim of the present study was to identify the variation in the concentration of fatty acids, according to different factors, among ten edible marine fish species in Mexico, collected from June to December 2009 in the largest fish market in Mexico city: Euthynnus alletteratus, Sciaenops ocellatus, Bairdiella chrysoura, Sphyraena guachancho, Symphurus elongatus, Istiophorus platypterus, Ophichthus rex, Eugerres plumieri, Eucinostomus entomelas and Oreochromis mossambicus. Lipid content was gravimetrically quantified, the fatty acids were determined using a gas chromatograph and the results were statistically analyzed. Total lipid content ranged from 0.93 to $1.95 \mathrm{~g} / 100 \mathrm{~g}$ in E. entomelas and $O$. urolepis hornorum, respectively. E. alletteratus, B. chrysoura, S. elongatus, I. platypterus, O. rex and E. plumieri presented the following order in FA concentration: Polyunsaturated FA (PUFA) $>$ Saturated FA (SFA) $>$ Monounsaturated FA (MUFA). S. ocellatus, S. guachancho and E. entomelas presented SFA $>$ PUFA $>$ MUFA; and only O. mossambicus presented SFA $>$ MUFA $>$ PUFA. O. mossambicus had the highest concentration (mg/100g) of SFA (559.40) and MUFA (442.60), while B. chrysoura presented the highest content (mg/100g) of PUFA (663.03), n-3 PUFA (514.03), EPA+DHA (506.10) and n-6 PUFA (145.80). Biotic and abiotic factors of the analyzed fish significantly influenced their FA concentration. Subtropical species presented $42.1 \%$ more EPA+DHA than tropical specie. Values presented here will vary according to the changes in the ecosystem and characteristics of each fish species, however the information generated in the present study is useful for improving fish consumption recommendations. Rev. Biol. Trop. 61 (4): 1981-1998. Epub 2013 December 01.
\end{abstract}

Key words: fatty acids, marine fish, biotic factors, abiotic factors, Mexico.

Los lípidos de la dieta influyen en el metabolismo, crecimiento y bienestar de los animales (Sargent, Bell, Bell, Henderson \& Tocher, 1993; Sargent, Bell, McEvoy, Tocher \& Estevez, 1999; Steffens, 1997). Se requieren para proveer energía y producir fosfolípidos y lípidos polares fundamentales para la formación de la membrana celular. En los peces, los lípidos son la principal fuente de energía para su crecimiento, especialmente durante el desarrollo embrionario; y son esenciales para su desarrollo neural, por lo que es común que en su cerebro y retina se encuentren altas concentraciones de ácido docosahexaenoico (C22:6n-3, DHA), ácido graso (AG) del tipo n-3 (Sargent et al., 1993). La ingesta de AG también determina el metabolismo de algunas enzimas que participan en la biosíntesis de los ácidos grasos endógenos en el caso de los peces de agua dulce, pues éstos son capaces de convertir algunos ácidos grasos poliinsaturados (AGP) (EPA (C20:5n-3, ácido 
eicosapentaenoico) y DHA) a partir de AG de cadena corta (ácido linolénico, por ejemplo); sin embargo las especies marinas son incapaces de hacerlo y su alimentación determina sus niveles de AGPn-3. La velocidad de conversión del EPA a DHA es menor en estos peces, lo que indica que estos ácidos son esenciales para las especies marinas. Tanto las especies dulceacuícolas como las marinas requieren pequeñas cantidades de AGn-6, principalmente C20:4n-6 (ácido araquidónico) para la formación de eicosanoides (Sargent et al., 1993; Sargent et al., 1999; Steffens, 1997). Existe gran variación en la composición lipídica de las especies de peces (Kris-Etherton et al., 2000; Molina de Salazar, 2009; Passi, Cataudella, Di Marco, De Simone \& Rastrelli, et al., 2002), y ésta depende de diversos factores bióticos y abióticos, como madurez, género, tamaño, estación, profundidad de las especies, entre otros (Ghaddar \& Saoud, 2012; Kris-Etherton et al., 2000; Patton, 1975; Tang, Chen, Xiao \& Wu, 2009). La temperatura del ambiente en el que viven las especies desempeña un papel fundamental en su perfil de AG. Aquellas que se han adaptado a bajas temperaturas $\left(5-10^{\circ} \mathrm{C}\right)$ presentan mayor concentración de ácidos grasos poliinsaturados que aquellas especies que se encuentran en un ambiente más cálido $\left(25-27^{\circ} \mathrm{C}\right)$ (Dey, Buda, Wiik, Halver \& Farkas, 1993; Lewis, 1962). Esta adaptación determina cuáles AG se presentan en mayor concentración. Las especies de ambientes fríos (tanto marinas como dulceacuícolas) tienen concentraciones más elevadas de ácido oleico y EPA, mientras que lo contrario sucede con el ácido araquidónico (Dey et al., 1993). La alimentación de los peces también es determinante para el perfil de sus AG, y su contenido de EPA y DHA puede atribuirse a la composición lipídica del plancton que consumen (Halver, 1980; Steffens, 1997). La salinidad del ambiente desempeña un papel importante en el perfil de ácidos grasos y por lo general, las especies de agua dulce presentan una mayor concentración de AGn-6 que las especies marinas, aunque el impacto que la salinidad del ambiente tiene sobre el contenido lipídico varía en distintas especies (Halver,
1980). La variación estacional asimismo ejerce cambios significativos en el contenido lipídico de los peces, y se ha observado una ligera tendencia a presentar mayor grado de insaturaciones en los AG durante la primavera (Halver, 1980). La etapa del ciclo de vida de los peces también determina su composición lipídica: durante la reproducción e incubación los peces deben incorporar ácidos grasos esenciales al embrión; los cuales varían según la especie (Halver, 1980). Todos los factores anteriores interactúan y determinan la composición de ácidos grasos de los peces.

Para el hombre, el pescado ha sido alimento desde hace muchos siglos, y el interés por estudiar la composición y beneficios nutrimentales de su consumo surgió desde hace varias décadas. Las especies de pescado tienen componentes nutrimentales necesarios en la dieta humana, especialmente los AG EPA y DHA (Tang et al., 2009). El EPA desempeña un papel cardioprotector en el cuerpo humano, mientras que el DHA interviene en el desarrollo de tejidos nerviosos, particularmente cerebro y retina (Valenzuela \& Videla, 2011). El consumo humano de estos AG aporta beneficios para la salud pues previenen el desarrollo de múltiples patologías, entre las que destaca la enfermedad cardiovascular y eventos coronarios (Brookhyser, 2006; Kris-Etherton, Harris, Lawrence \& Nutrition Comittee, 2002; Vrablík, Prusíková, Snejdrlová \& Zlatohlávek, 2009); también existe evidencia clínica de su participación benéfica en enfermedades con inflamación crónica (University of Maryland, 2011; Wall, Ross, Fitzgerald \& Stanton, 2010); se ha encontrado asociación entre su consumo y la disminución en la aparición de depresión y desórdenes mentales (Hedelin et al., 2010; Sánchez-Villegas, Henríquez, Figueiras, Ortuño, Lahortiga \& Martínez-González, 2007), además de que se sugiere que protegen contra lesiones cardiacas y hepáticas asociadas a isquemia (Valenzuela et al., 2011).

Al considerar dichos beneficios, las recomendaciones actuales sobre su consumo dadas por la Asociación Americana del Corazón (American Heart Association), sugieren incluir 
pescado en la dieta cuando menos dos veces por semana (Gidding et al., 2009; Kris-Etherton et al., 2002; Lichtenstein et al., 2006). Los estudios sobre la composición nutrimental de especies marinas de consumo humano permiten conocer las propiedades alimenticias de los peces, y generan información útil para fines terapéuticos y nutricionales (D’Armas, Yáñez, Reyes \& Salazar, 2010). Debido al creciente interés en el contenido nutrimental de los peces para una óptima selección de los mismos, el objetivo del presente trabajo fue caracterizar el perfil de ácidos grasos en diez especies de pescados consumidas en México, así como su variación dada por factores bióticos (especie) y abióticos (clima, distribución batimétrica y origen).

\section{MATERIALES Y MÉTODOS}

Obtención de muestras: Las diez especies de pescado analizadas se obtuvieron de La Nueva Viga, el centro de distribución de recursos pesqueros más grande de América Latina, ubicado en la Ciudad de México (Castro-González, Ojeda, Montaño, Ledesma \& Pérez-Gil, 2007), entre junio y diciembre de 2009. Se colectaron al azar 15 pescados frescos de cada una de las diez especies, provenientes de distintas bodegas del mercado. Los pescados se filetearon, se colocaron en contenedores térmicos y se trasladaron al laboratorio, en donde fueron congelados a $-4^{\circ} \mathrm{C}$ para su posterior análisis. Entre la recolección y el análisis de todas las especies transcurrieron cinco días. Se guardó un pescado entero para su identificación taxonómica.

Identificación de las especies: Todas las especies analizadas son de consumo humano y se comercializan en los mercados de la ciudad. En su identificación se utilizaron fotografías, claves taxonómicas, características morfológicas y bases de datos de peces (Froese \& Pauly, 2011). En el cuadro 1 se presentan nombres comunes en español, nombre en inglés y nombre científico de las especies analizadas.
Preparación de las muestras: Un total de $2 \mathrm{~kg}$ de filetes crudos y sin espinas de cada especie, se molieron y homogeneizaron para obtener una submuestra; de la cual se obtuvieron $100 \mathrm{~g}$ al azar para los análisis químicos. Previamente se agregó a las muestras butilhidroxitolueno (BHT) J.T. Baker como antioxidante, en una concentración de $0.01 \%$ (Pérez, 2000).

Determinación de los lípidos totales: Estos nutrimentos se cuantificaron de acuerdo a la técnica de Folch, Lees \& Stanley (1957) modificada según el siguiente procedimiento: Se pesó $1 \mathrm{~g}$ de muestra y se homogeneizó al añadir una solución de cloroformo (99\% cloroformo y $1 \%$ etanol como preservativo) y metanol puro (J.T. Baker) 2:1 hasta tener un volumen de $20 \mathrm{~mL}$. La mezcla se agitó por $2 \mathrm{hr}$ a temperatura ambiente en un agitador orbital a 500rpm. La mezcla con la muestra se filtró con papel filtro doblado Whatman núm. 4 y se enjuagó tres veces con una mezcla de metanol (J.T. Baker) y agua 1:1. La fase orgánica se evaporó bajo flujo de nitrógeno gaseoso (Infra nitrógeno grado USP-NF UN 1066); una vez fría y seca la muestra, el contenido lipídico se calculó gravimétricamente (Association of Official Analytical Chemists, 1996; Blight \& Dyer, 1959; Folch et al., 1957; Morrison \& Smith, 1964).

Determinación de ácidos grasos: $\mathrm{Se}$ procedió a una saponificación con potasa metanólica saturada para la obtención de los ácidos grasos libres, los cuales se esterificaron y metilaron con una solución de trifluoruro de boro-metanol al 14\% (J.T. Baker). Se evaporaron a sequedad en atmósfera de $\mathrm{N}_{2}$. La detección de AG se llevó a cabo con un cromatógrafo de gases Varian $3400 \mathrm{CX}$, equipado con una columna Supelco SP 2560 de $100 \times 0.25 \times 20 \mu \mathrm{m}$ y con detector de ionización de llama. La temperatura del inyector fue $250^{\circ} \mathrm{C}$ y la del detector $300^{\circ} \mathrm{C}$. La temperatura inicial de $140^{\circ} \mathrm{C}$ se incrementó $10^{\circ} \mathrm{C} / \mathrm{min}$ hasta $\operatorname{los} 200^{\circ} \mathrm{C}$. Esta temperatura se mantuvo por $1 \mathrm{~min}$, para luego incrementar $5^{\circ} \mathrm{C} / \mathrm{min}$ hasta 


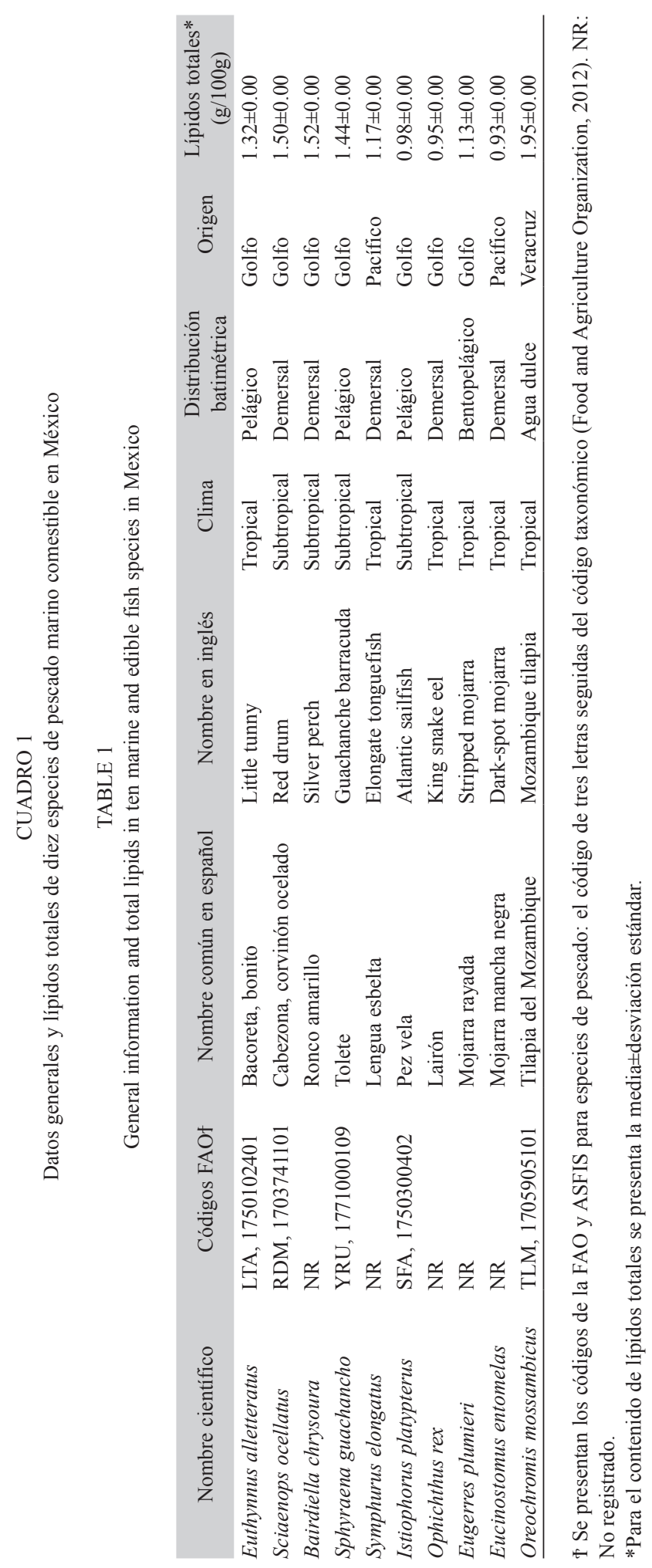


alcanzar los $270^{\circ} \mathrm{C}$, temperatura que se mantuvo por $7 \mathrm{~min}$. El volumen de inyección fue de $1 \mu \mathrm{L}$ para cada muestra. El estándar interno utilizado fue ácido miristoleico.

Identificación de los ácidos grasos: Se compararon los tiempos de retención de cada ácido graso con aquellos de la mezcla Supelco 37 FAME (fatty acid metyl esters). El contenido de ácidos grasos se calculó mediante las siguientes fórmulas:

1. Ecuación para la concentración estándar de ácidos grasos:

$$
\mathrm{FR}=(\mathrm{CPI} \times \mathrm{AEI}) /(\mathrm{API} \times \mathrm{CEI}),
$$

Donde FR es Factor de Respuesta; CPI es la Concentración del Pico de Interés; AEI es el Área del Estándar Interno; API es el Área del Pico de Interés; y CEI es la Concentración del Estándar Interno.

2. Ecuación de la concentración de ácidos grasos de la muestra:

$$
\text { CAGIm=(APIm x FR) / [(CEIM) / (AEIM) }] \text {, }
$$

Donde CAGIm es la Concentración del Ácido Graso de Interés de la muestra; APIm es Área del Pico de Interés de la muestra; CEIm es la Concentración del Estándar Interno de la muestra: y AEIm es el Área del Estándar Interno de la muestra.

En los resultados de los cuadros 2 y 3 se presenta la media de cinco repeticiones en $\mathrm{mg} / 100 \mathrm{~g}$ de filete, de acuerdo a la siguiente ecuación:

\section{CAGIM en $\mathrm{mg} / 100 \mathrm{~g}=(\mathrm{CAGIm} / \mathrm{MM}) \times 100$,}

donde MM es la Masa de la Muestra.

Los resultados de los lípidos totales (LT), ácidos grasos saturados (AGS), ácidos grasos monoinsaturados (AGM), ácidos grasos poliinsaturados (AGP) y ácidos grasos n-3 (AGn-3), ácidos grasos n-6 (AGn-6) y EPA+DHA se agruparon según sus factores abióticos en las siguientes categorías:

- Distribución batimétrica: Demersal, bentopelágico y pelágico; excepto la tilapia mosambica, que es de agua dulce.

- Clima: Tropical y subtropical.

- Origen: Golfo de México y Océano Pacífico; excepto la tilapia mosambica, que es del Estado de Veracruz.

Los LT se sometieron a un análisis descriptivo (media, desviación estándar, máximos y mínimos), y para la diferencia de medias de los grupos según su distribución batimétrica se aplicó un Análisis de varianza de una vía; mientras que para la diferencia de medias por clima y origen se aplicó una prueba de $\mathrm{t}$ de student, se utilizó el software SigmaPlot2008 para Windows.

Los AGS, AGM, AGP, AGn-3, AGn-6 y EPA+DHA se sometieron a un análisis descriptivo: media, desviación estándar, máximos y mínimos, sesgo y curtosis. Para las concentraciones de AGn-6 y EPA+DHA se aplicó un análisis de varianza. En los casos en que no fue razonable el supuesto de normalidad se aplicó un análisis de varianza no paramétrico (Kruskal-Wallis). Posteriormente se hicieron todas las comparaciones entre pares de especies ajustando la significancia por comparaciones múltiples con el método de Holm-Sidak o de Dunn. (Systat Software, 2008). Para presentar de manera gráfica el total de las concentraciones de AGS, AGM, AGP, AGn-6 y EPA+DHA por especie, se construyeron gráficas de radar con el software Stata10.0 2007 de Windows.

\section{RESULTADOS}

En el cuadro 1 se enlistan los nombres de las especies analizadas y las categorías abióticas a las que pertenecen.

Lípidos totales: Por su contenido de LT (Cuadro 1) todas las especies analizadas tuvieron $<2 \%$ de LT. En las especies tropicales el 
CUADRO 2

Ácidos grasos de seis especies de peces tropicales de importancia comercial (mg/100g)

TABLE 2

Fatty acids of six tropical fish species of commercial importance $(\mathrm{mg} / 100 \mathrm{~g})$

\begin{tabular}{|c|c|c|c|c|c|c|}
\hline Ácidos grasos & $\begin{array}{l}\text { Euthynnus } \\
\text { alleteratus* }\end{array}$ & $\begin{array}{l}\text { Oreochromis } \\
\text { mossambicus* }\end{array}$ & $\begin{array}{l}\text { Symphurus } \\
\text { elongatus* }\end{array}$ & $\begin{array}{l}\text { Ophichthus } \\
\text { rex* }\end{array}$ & $\begin{array}{l}\text { Eugerres } \\
\text { plumieri* }^{*}\end{array}$ & $\begin{array}{c}\text { Eucinostomus } \\
\text { entomelas* }\end{array}$ \\
\hline C14:0 & 9.10 & 47.00 & 3.00 & 6.80 & 5.10 & 16.90 \\
\hline C15:0 & 3.00 & 4.40 & 3.00 & 5.90 & 1.50 & 2.50 \\
\hline $\mathrm{C} 16: 0$ & 117.30 & 402.00 & 104.30 & 72.50 & 72.20 & 124.10 \\
\hline $\mathrm{C} 17: 0$ & 8.10 & 15.80 & 7.20 & 4.60 & 4.20 & 7.30 \\
\hline C18:0 & 75.30 & 68.30 & 51.00 & 32.10 & 33.80 & 40.10 \\
\hline C20:0 & 4.90 & 6.90 & 0.90 & 1.20 & 1.40 & 0.50 \\
\hline $\mathrm{C} 21: 0$ & 3.80 & 10.60 & ND & 0.40 & 0.30 & 4.30 \\
\hline $\mathrm{C} 23: 0$ & 1.10 & 1.10 & 3.10 & 0.60 & ND & 0.30 \\
\hline $\mathrm{C} 24: 0$ & 5.10 & 3.30 & 0.80 & 0.40 & ND & 0.70 \\
\hline Total AGS & 227.70 & 559.40 & 173.30 & 124.50 & 118.50 & 196.70 \\
\hline C15:1 & 1.30 & 1.20 & 3.40 & 26.30 & 21.30 & 3.90 \\
\hline C16:1 n-9 & 12.10 & 114.50 & 9.70 & 20.20 & 8.30 & 21.10 \\
\hline C17:1 & 2.40 & 5.00 & 4.50 & 4.50 & 2.70 & 1.80 \\
\hline $\mathrm{C} 18: 1 \mathrm{n}-9 \mathrm{t}$ & 32.40 & 3.80 & 0.01 & 1.20 & 1.90 & 1.10 \\
\hline C18:1 n-9 & 36.80 & 278.50 & 148.80 & 44.20 & 52.70 & 81.60 \\
\hline C20:1 n-9 & 2.00 & 16.90 & 6.40 & 2.10 & 1.50 & 3.10 \\
\hline C22:1 n-9 & 5.60 & 2.10 & ND & 0.20 & 1.40 & ND \\
\hline Total AGM & 92.60 & 422.60 & 172.81 & 98.70 & 89.80 & 112.60 \\
\hline $\mathrm{C} 18: 2 \mathrm{n}-6 \mathrm{t}$ & 0.30 & 1.20 & 1.77 & 0.44 & 0.20 & 1.20 \\
\hline C18:2 n-6 & 9.40 & 60.60 & 7.60 & 3.80 & 9.80 & 15.70 \\
\hline C18:3 n-6 & 1.70 & 21.90 & 1.90 & 1.90 & 3.20 & 1.12 \\
\hline C18:3 n-3 & 3.23 & 81.78 & 1.20 & 1.00 & 0.95 & 13.30 \\
\hline$C 20: 2 n-6$ & 3.30 & 4.90 & 1.90 & 1.30 & 1.80 & 1.60 \\
\hline$C 20: 3 n-3$ & 1.96 & 1.17 & ND & 0.19 & 0.70 & ND \\
\hline$C 20: 3 n-6$ & 2.00 & 14.90 & 0.82 & 1.03 & 1.70 & 2.60 \\
\hline C20:4 n-6 & 39.40 & 37.60 & 49.50 & 32.60 & 43.00 & 23.00 \\
\hline$C 20: 5 n-3$ & 39.94 & 21.33 & 33.30 & 16.81 & 29.14 & 8.90 \\
\hline$C 22: 2$ n-6 & ND & 2.16 & ND & ND & 1.20 & ND \\
\hline $\mathrm{C} 22: 6 \mathrm{n}-3$ & 175.72 & 64.37 & 202.10 & 91.37 & 43.55 & 70.80 \\
\hline Total AGP & 276.95 & 311.91 & 300.09 & 150.44 & 135.24 & 138.22 \\
\hline $\mathrm{EPA}+\mathrm{DHA}$ & 215.60 & 85.70 & 235.40 & 108.20 & 72.70 & 79.70 \\
\hline AG n-3 & 220.85 & 168.65 & 236.60 & 109.37 & 74.34 & 93.00 \\
\hline AG n-6 & 56.10 & 143.26 & 63.49 & 41.07 & 60.90 & 45.22 \\
\hline$n-6 / n-3$ & 0.25 & 0.84 & 0.26 & 0.37 & 0.81 & 0.48 \\
\hline
\end{tabular}

ND: No detectado, AGS: Ácidos grasos saturados, AGM: Ácidos grasos monoinsaturados; AGP: Ácidos grasos poliinsaturados, EPA: Ácido eicosapentaenóico, DHA: Ácido docosahexaenoico, AG n-3: Ácidos grasos n-3, AG n-6: Ácidos grasos n-6.

*Se presenta la media de cinco repeticiones. 
CUADRO 3

Ácidos grasos de cuatro de especies de peces marinos subtropicales de importancia comercial (mg/100g)

TABLE 3

Fatty acids of four subtropical fish species of commercial importance $(\mathrm{mg} / 100 \mathrm{~g})$

\begin{tabular}{|c|c|c|c|c|}
\hline Ácidos grasos & $\begin{array}{l}\text { Sciaenops } \\
\text { ocellatus * }\end{array}$ & $\begin{array}{l}\text { Bairdiella } \\
\text { chrysoura* }\end{array}$ & $\begin{array}{c}\text { Sphyraena } \\
\text { guachancho* }\end{array}$ & $\begin{array}{l}\text { Istiophorus } \\
\text { platypterus* }\end{array}$ \\
\hline C14:0 & 9.80 & 8.20 & 16.40 & 1.90 \\
\hline $\mathrm{C} 15: 0$ & 4.40 & 3.30 & 4.40 & 1.10 \\
\hline C16:0 & 189.10 & 177.60 & 191.40 & 44.90 \\
\hline $\mathrm{C} 17: 0$ & 8.30 & 11.80 & 8.60 & 0.20 \\
\hline C18:0 & 68.90 & 99.20 & 59.90 & 32.80 \\
\hline C20:0 & 2.10 & 2.70 & 3.20 & 0.50 \\
\hline $\mathrm{C} 21: 0$ & 0.30 & ND & 0.50 & 0.30 \\
\hline C23:0 & 0.80 & ND & 1.10 & ND \\
\hline C24:0 & ND & ND & 2.30 & 0.30 \\
\hline Total AGS & 283.70 & 302.80 & 287.80 & 82.00 \\
\hline C15:1 & 2.90 & 2.60 & 1.70 & 0.40 \\
\hline C16:1 n-9 & 47.60 & 17.60 & 35.80 & 3.40 \\
\hline C17:1 & 5.80 & 4.90 & 4.70 & 1.30 \\
\hline C18:1 n-9t & 2.40 & ND & 0.90 & 0.30 \\
\hline $\mathrm{C} 18: 1 \mathrm{n}-9$ & 186.40 & 100.90 & 102.30 & 24.00 \\
\hline C20:1 n-9 & 4.30 & 3.00 & 3.20 & 0.70 \\
\hline C22:1 n-9 & ND & 1.50 & 0.60 & ND \\
\hline Total AGM & 249.40 & 130.50 & 149.20 & 30.10 \\
\hline $\mathrm{C} 18: 2 \mathrm{n}-6 \mathrm{t}$ & 0.60 & 3.20 & 0.60 & 0.30 \\
\hline $\mathrm{C} 18: 2 \mathrm{n}-6$ & 38.80 & 13.40 & 9.60 & 5.80 \\
\hline C18:3 n-6 & 1.00 & 6.40 & 2.20 & 1.90 \\
\hline C18:3 n-3 & 3.40 & 2.10 & 2.81 & 0.61 \\
\hline$C 20: 2$ n-6 & 3.00 & 2.60 & 2.20 & 1.20 \\
\hline$C 20: 3 n-3$ & 0.90 & 5.44 & 0.64 & 0.12 \\
\hline C20:3 n-6 & 3.80 & 2.70 & 1.80 & 0.80 \\
\hline C20:4 n-6 & 82.90 & 119.30 & 41.80 & 24.20 \\
\hline$C 20: 5 n-3$ & 42.38 & 80.43 & 30.05 & 11.53 \\
\hline C22:2 n-6 & ND & 1.40 & 0.70 & ND \\
\hline$C 22: 6 n-3$ & 85.79 & 426.06 & 149.33 & 92.42 \\
\hline Total AGP & 262.57 & 663.03 & 241.74 & 138.88 \\
\hline EPA+DHA & 128.20 & 506.10 & 179.40 & 103.90 \\
\hline AG n-3 & 132.47 & 514.03 & 182.83 & 104.68 \\
\hline AG n-6 & 130.10 & 145.80 & 58.91 & 34.20 \\
\hline$n-6 / n-3$ & 0.98 & 0.28 & 0.32 & 0.32 \\
\hline
\end{tabular}

ND: No detectado, AGS: Ácidos grasos saturados, AGM: Ácidos grasos monoinsaturados; AGP: Ácidos grasos poliinsaturados, EPA: Ácido eicosapentaenóico, DHA: Ácido docosahexaenoico, AG n-3: Ácidos grasos n-3, AG n-6: Ácidos grasos n-6.

*Se presenta la media de cinco repeticiones. 
contenido varió desde $0.93 \mathrm{~g} / 100 \mathrm{~g}$ de $\mathrm{LT}$ en la mojarra mancha negra, hasta $1.95 \mathrm{~g} / 100 \mathrm{~g}$ en la tilapia del Mozambique; mientras que en las especies subtropicales el menor contenido lo presentó el pez vela $(0.98 \mathrm{~g} / 100 \mathrm{~g})$ y la mayor concentración se observó en el ronco amarillo $(1.52 \mathrm{~g} / 100 \mathrm{~g})$. No se encontraron diferencias significativas entre los grupos según las categorías.

Ácidos grasos saturados: En los cuadros 2 y 3 se presenta el perfil de ácidos grasos de las diferentes especies agrupadas por su distribución climática. Los AGS identificados fueron nueve. Los ácidos C21:0, C23:0 y C24:0 no se identificaron en todas las especies. El más abundante en todas las especies fue el ácido palmítico (C16:0), con valores desde 72.20 hasta $402.00 \mathrm{mg} / 100 \mathrm{~g}$ en las especies tropicales y 44.90 hasta $191.40 \mathrm{mg} / 100 \mathrm{~g}$ en las especies subtropicales. El 50\% de especies presentó un contenido $<200 \mathrm{mg} / 100 \mathrm{~g}$ y el $30 \%$ tuvo entre 250 y $303 \mathrm{mg} / 100 \mathrm{~g}$.

En la figura 1A se presentan la media y desviación estándar de los AGS de las especies analizadas. La tilapia del Mozambique fue la especie con mayor concentración y sólo fue diferente significativamente (Dunn, $\mathrm{p}<0.05$ ) con el pez vela, el cual presentó la menor concentración de AGS.

En el cuadro 4 se presenta la variación del total de AGS de las especies, de acuerdo a las clasificaciones de origen, clima y distribución batimétrica.

Ácidos grasos monoinsaturados: Se identificaron siete ácidos grasos monoinsaturados. No se identificaron ni el ácido C18:1n-9t ni C22:1n-9 en todas las especies. El ácido que se encontró en mayor cantidad en todas las especies fue el oleico (C18:1n-9), desde 36.80 hasta $278.50 \mathrm{mg} / 100 \mathrm{~g}$ en las especies tropicales; y desde 24.00 hasta $186.40 \mathrm{mg} / 100 \mathrm{~g}$ en las especies subtropicales. El $40 \%$ de los peces
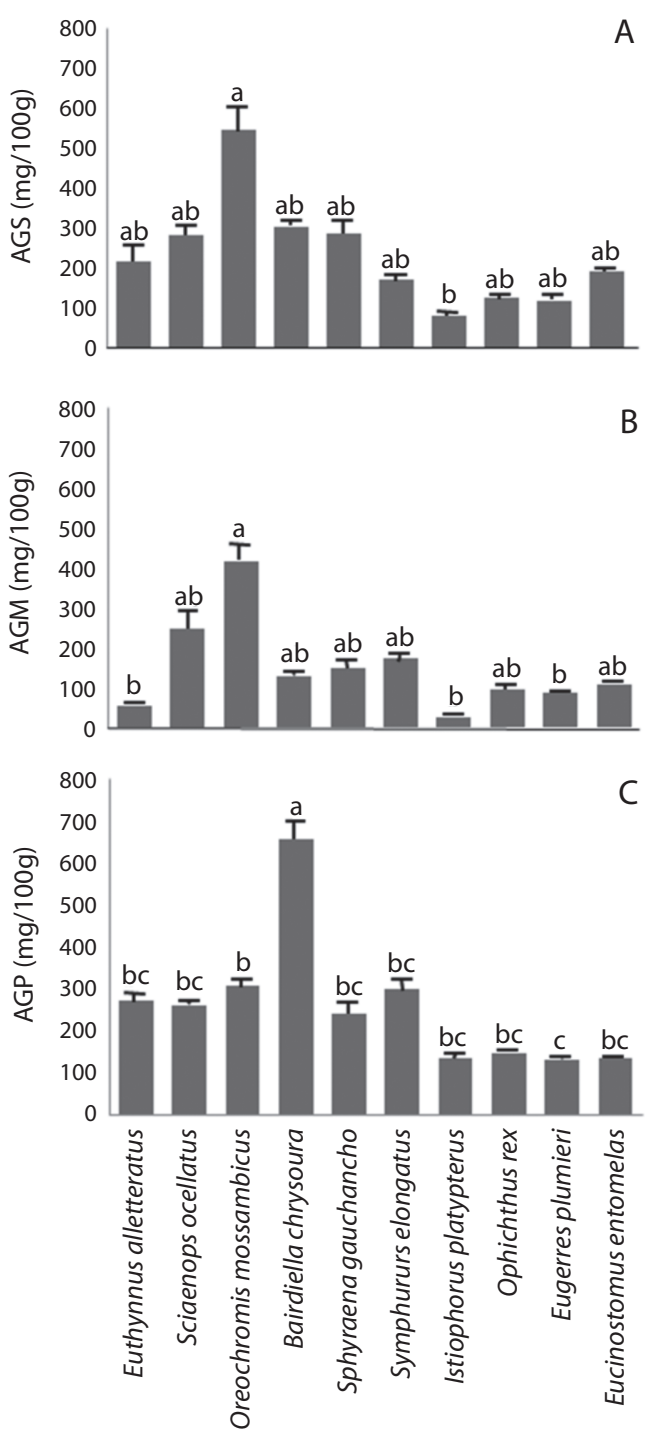

Fig. 1. Media y desviación estándar, de las especies analizadas, para (A) Ácidos grasos saturados (AGS), (B) Ácidos grasos monoinsaturados (AGM), y (C) Ácidos grasos poliinsaturados (AGP). a, b, c. Literales distintas indican diferencias significativas (Dunn, $\mathrm{p}<0.05$ para AGS y AGM; y Holm-Sidak, $\mathrm{p}<0.001$ para AGP).

Fig. 1. Mean and standard deviation of the analyzed species for (A) Saturated fatty acids (AGS), (B) Monounsaturated fatty acids (AGM), and (C) Polyunsaturated fatty acids (AGP). a, b, c. Different letters indicate significant differences (Dunn, $\mathrm{p}<0.05$ for AGS and AGM; and HolmSidak, $\mathrm{p}<0.001$ for AGP). 


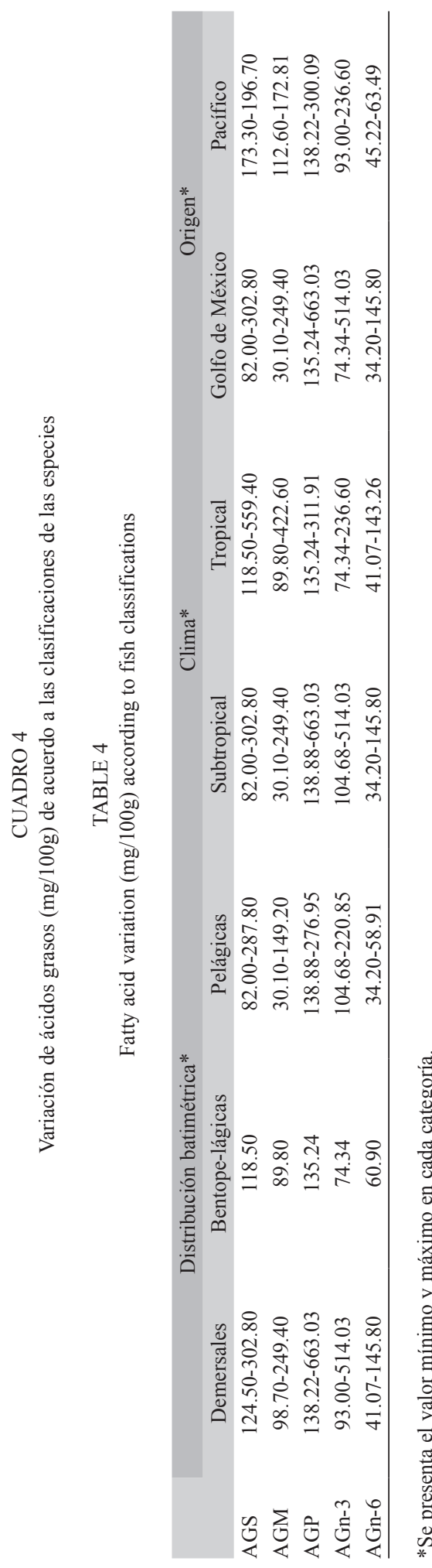

presentó valores $<100 \mathrm{mg} / 100 \mathrm{~g}$; y el $30 \%$ tuvo concentraciones $>150 \mathrm{mg} / 100 \mathrm{~g}$.

En la Fig. 1B se presentan la media y desviación estándar de los AGM de las especies analizadas. Se observa que la tilapia mosambica fue el pescado con mayor número de diferencias significativas (Dunn, $\mathrm{p}<0.05$ ). De acuerdo con los AGM de las especies y su distribución climática, se encontraron diferencias significativas en dos casos: entre una especie tropical (bonito) vs. otra tropical (lengua), y entre una especie tropical (lengua) vs. una subtropical (vela).

En el cuadro 4 se presenta la variación del total de AGM de las especies, de acuerdo a las clasificaciones de origen, clima y distribución batimétrica.

Ácidos grasos poliinsaturados: Se identificaron 11 AGP. No se identificaron los ácidos grasos C20:3n-3 ni C22:2n-6 en todas las especies. El más abundante en todos los peces fue el C22:6n-3 (DHA), excepto en la tilapia del Mozambique que fue el C18:3n-3 (ácido $\alpha$-linolénico). El 40\% de los peces analizados tuvo valores $<200 \mathrm{mg} / 100 \mathrm{~g}$; y en el $60 \%$ de las especies se encontraron concentraciones $>240 \mathrm{mg} / 100 \mathrm{~g}$.

En el cuadro 4 se presenta la variación del total de AGP de las especies, de acuerdo a las clasificaciones de origen, clima y distribución batimétrica.

En la figura 1C se presentan los AGP de las especies analizadas. Se hicieron todas las comparaciones múltiples entre pares de especies con el método de Holm-Sidak o de Dunn, según sea el caso. Con los resultados obtenidos se identificaron las diferencias significativas entre las especies y éstas se clasificaron de acuerdo a sus factores abióticos:

1. Distribución batimétrica: Cuatro diferencias significativas entre especies demersales vs. demersales, tres entre demersales vs. pelágicas y una entre demersales vs. bentopelágicas.

2. Distribución climática: Se encontraron seis diferencias significativas entre tropicales 
vs. subtropicales, tres entre subtropicales

vs. subtropicales y una entre tropicales vs. tropicales.

3. Origen: Se encontraron seis diferencias significativas entre especies del Golfo de México vs. Golfo de México, y dos entre Golfo de México vs. Océano Pacífico.

En la figura 2A se muestra la concentración total de AGS, AGM y AGP en cada una de las especies analizadas; se observa que en todas, excepto en el ronco amarillo y la tilapia del Mozambique, existe una notable agrupación de los AGS, AGM y AGP por debajo del perímetro de 280.

Las especies analizadas presentaron una mayor concentración total de AGS en comparación con AGM. Seis especies (el ronco amarillo, el pez vela, la bacoreta, la lengua esbelta, el lairón y la mojarra rayada) presentaron la concentración de ácidos grasos en el siguiente orden: AGP>AGS>AGM. El corviñón ocelado, el tolete y la mojarra mancha negra presentaron el siguiente orden: AGS $>$ AGP $>$ AGM; mientras que tan sólo la tilapia del Mozambique presentó una concentración de AGS $>$ AGM $>$ AGP.

Ácidos grasos n-3 y n-6: Se identificaron cuatro AGn-3. El ácido C20:3n-3 no fue identificado en la lengua esbelta y la mojarra mancha negra. La concentración de AGn-3 en las especies analizadas varió desde 13.03 hasta $47.02 \%$ AGT, la variación del total de AGn-3 de las especies de acuerdo a las clasificaciones dadas por los factores abióticos se presentan en el cuadro 4.

Se identificaron siete AGn-6, y el C22:2n-6 no fue identificado en todos los peces. Únicamente tres especies tuvieron valores $>70 \mathrm{mg}$ AGn-6/100g. La concentración de AGn-6 en las especies analizadas varió de 8.6 a $13.63 \%$ AGT. En el cuadro 4 se presenta la variación del total de AGn-6 de las especies, de acuerdo a las clasificaciones de origen, clima y distribución batimétrica.

En la figura $3 \mathrm{~A}$ se presentan la media y desviación estándar de los AGn-6 de las especies estudiadas. Analizando las categorías de los factores abióticos se encontraron las siguientes diferencias estadísticas (HolmSidak, $\mathrm{p}<0.001$ ):

1. Distribución batimétrica: Se encontraron tres diferencias significativas entre pelágicas vs. demersales, dos entre demersales vs. demersales y una entre bentopelágicas vs. demersales.
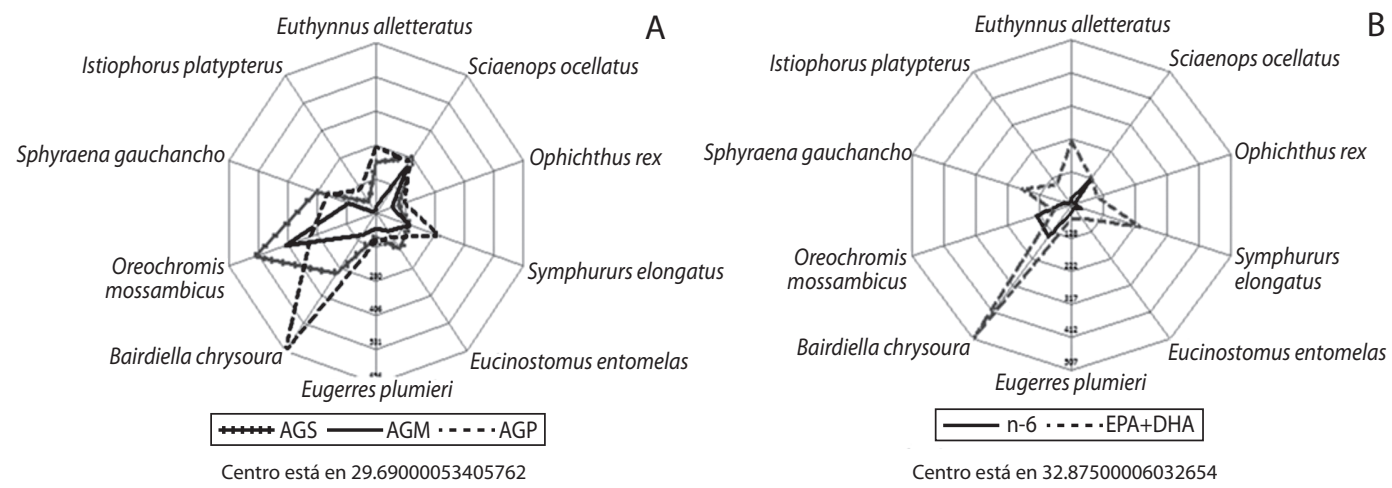

Fig. 2. (A) Concentración del total de ácidos grasos saturados (AGS), ácidos grasos monoinsaturados (AGM) y ácidos grasos poliinsaturados (AGP) en las diez especies analizadas. (B) Contenido del total de AG n-6 y ácidos eicosapentaenoic o+docosahexaenoico (EPA+DHA) en las diez especies de pescado.

Fig. 2. (A) Total saturated fatty acids (AGS), Monounsaturated fatty acids (AGM) and Polyunsaturated fatty acids (AGP) of the ten analyzed species. (B) Total AG n-6 and eicosapentaenoic+docosahexaenoic acids (EPA+DHA) content in the analyzed species. 

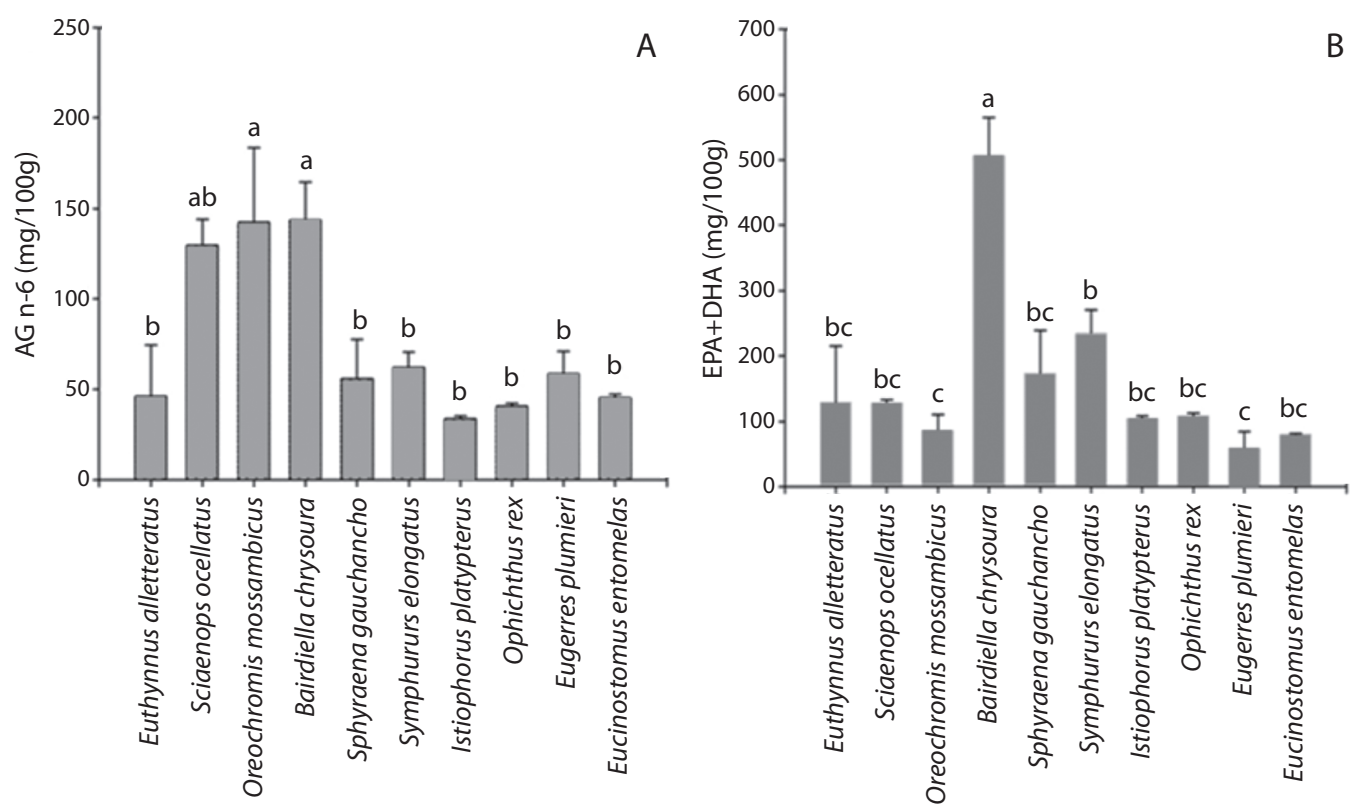

Fig. 3. Media y desviación estándar, de las especies analizadas, para (A) Ácidos grasos poliinsaturados n-6 (AGP n-6), (B) Ácido eicosapentaenoico+ácido docosahexaenoico (EPA+DHA). a, b, c. Literales distintas indican diferencias significativas (Holm-Sidak, $\mathrm{p}<0.001)$.

Fig. 3. Mean and standard deviation of the analyzed species for (A) n-6 polyunsaturated fatty acids (AGP n-6), (B) Eicosapentaenoic acid+docosahexaenoico acid (EPA+DHA). a, b, c. Different letters indicate significant differences (HolmSidak, $\mathrm{p}<0.001)$.

2. Distribución climática: Se encontraron seis diferencias significativas entre especies tropicales vs. subtropicales, cinco entre tropicales vs. tropicales y dos entre subtropicales vs. subtropicales.

3. Origen: Se encontraron cinco diferencias significativas entre especies del Golfo de México vs. Golfo de México y una entre Golfo de México y Océano Pacífico.

Con los valores de AGn-3 y n-6 se calculó la relación n-6/n-3 de las especies estudiadas y se observó que todas las especies tuvieron una relación $<1$, desde 0.25 a 0.98 .

La concentración de EPA+DHA varió de la siguiente manera: En las especies tropicales, la lengua esbelta presentó la mayor concentración mientras que en las subtropicales fue en el ronco amarillo. En las especies subtropicales se observó mayor concentración, con valores por encima de $100 \mathrm{mg} / 100 \mathrm{~g}$ de filete. Tan sólo el $30 \%$ de todas las especies presentó valores por debajo de 100mg EPA+DHA/100g, pertenecientes todas a los peces tropicales. En el presente estudio el intervalo de EPA fue de 8.90 a $42.38 \mathrm{mg} / 100 \mathrm{~g}$, excepto en el ronco amarillo. El DHA se presentó en una concentración de 43.55 a $426.06 \mathrm{mg} / 100 \mathrm{~g}$.

El contenido de EPA+DHA en los pescados analizados fue muy variable entre las especies (Cuadros 2 y 3), con un intervalo desde 72.70 hasta $506.10 \mathrm{mg} / 100 \mathrm{~g}$.

En la figura $3 \mathrm{~B}$ se presentan la media y desviación estándar de EPA+DHA. Analizando las categorías de los factores abióticos se encontraron las siguientes diferencias estadísticas (Holm-Sidak, $\mathrm{p}<0.001)$ :

1. Por su distribución batimétrica: Dos entre especies demersales vs. bentopelágicas, cuatro entre demersales vs. demersales y tres entre demersales vs. pelágicas. 
2. Por su origen: Seis especies del Golfo de México vs. Golfo de México y tres entre Golfo de México vs. Océano Pacífico.

3. Por su distribución climática: Seis entre especies tropicales vs. subtropicales, tres entre subtropicales vs. subtropicales, y dos entre tropicales vs. tropicales.

En la figura $2 \mathrm{~B}$ se presenta la concentración de AGn-6 y EPA+DHA en cada una de las especies analizadas. Existe una clara diferencia entre el comportamiento de los AGn-6 y EPA+DHA.

\section{DISCUSIÓN}

Lípidos totales (LT): Por su contenido de lípidos totales, según la clasificación de especies de pescado de consumo humano, todas las especies analizadas fueron magras $(<2 \%$ de LT) (Nurnadia, Azrina \& Amin, 2011). Los resultados son semejantes a los encontrados por Rasoarahona, Barnathan, Bianchini \& Gaydou (2005) en tres especies de la tilapia, de 0.83 a $1.29 \mathrm{~g} / 100 \mathrm{~g}$; por Romero, Robert, Masson \& Pineda (2000) en siete especies de pescados marinos, desde 0.3 hasta $3.6 \mathrm{~g} / 100 \mathrm{~g}$ y por Saito, Yamashiro, Alasalvar \& Konno (1999) en tres especies subtropicales (0.6 a $0.8 \mathrm{~g} / 100 \mathrm{~g}$ ). En otros estudios los LT presentaron una gran variación, tal es el caso del salmón (7.4-17.0g/100g) analizado por Perea, Gómez, Mayorga \& Triana (2008); mientras que Özogul, Özogul, Cícek, Polat \& Kuley (2009) encontraron una variación desde 0.3 hasta $10.67 \mathrm{~g} / 100 \mathrm{~g}$ en 34 especies. La cantidad de LT depende de factores bióticos como la especie, el estado fisiológico, edad, sexo (Huynh, Kitts, Hu \& Trites, 2007; Özogul et al., 2009; Passi et al., 2002; Usydus, Szlifder-Richert \& Adamcdzyk, 2012), así como factores abióticos: temperatura, salinidad, profundidad, origen geográfico, época de captura, etc. (Njinkoué, Barnathan, Miralles, Gaydou \& Samb, 2002; Özogul et al., 2009; Saito et al., 1999; Tanakol, Yazici, Sener \& Sencer, 1999).

Los LT, además de AG, se componen de otros elementos; sin embargo, se observa una tendencia a presentar mayor concentración de ácidos grasos mientras mayor sea el contenido de LT en las especies analizadas, lo cual corresponde a lo presentado en el estudio de Perea et al. (2008). Los valores reportados en el presente estudio pueden estar relacionados con las condiciones ambientales al momento de captura de cada especie (D’Armas et al., 2010).

Ácidos grasos saturados (AGS): En el presente estudio no se identificaron los ácidos grasos saturados (AGS) C15:0iso, C17:0iso y C22:0, los cuales fueron detectados en especies de Senegal, del Mar Negro, del Mar de Marmara, del mar del este de China y de la Isla de Pascua en Chile (Huynh et al., 2007; Njinkoué et al., 2002; Romero et al., 2000; Saito et al., 1999; Tanakol et al., 1999).

El AGS más abundante en los estudios revisados (Huynh et al., 2007; Njinkoué et al., 2002; Özogul et al., 2009; Passi et al., 2002; Romero et al., 2000; Tanakol et al., 1999) fue el palmítico (C16:0), con un valor mínimo en porcentaje de AG totales (\%AGT) de 7.4 (Phyllonothus trunculus), (Passi et al., 2002) y un valor máximo de 44.3 (Bodianus vulpinus) (Romero et al., 2000). El ácido palmítico es reconocido como una fuente de energía metabólica durante el crecimiento y formación de hueva en los peces (Huynh et al., 2007).

Otro AGS con una amplia variación en su concentración es el C14:0 (ácido mirístico). En el presente trabajo los valores de este ácido variaron desde $0.47 \%$ del total de AG en la lengua esbelta hasta $3.80 \%$ en la mojarra mancha negra, mientras que Ortiz \& Bosch (1994), lo identificaron como el segundo AGS más abundante en el bonito y la sardina con valores de 11.3 y $10.4 \%$, respectivamente. Tanakol et al. (1999) lo encontraron con porcentajes de 7.1 en la anchoveta y $7.4 \%$ en la sardina. Otro AGS abundante es el ácido esteárico (C18:0), cuyo intervalo en el presente estudio fue de $5.3 \%$ (en la tilapia mosambica) hasta $13.1 \%$ (el pez vela); situándose como el segundo AGS más abundante; Tanakol et al. (1999), reportaron un contenido de 3.5 a $7.8 \%$ en especies del Mar Negro y Mar Marmara. 
El contenido lipídico y el perfil de AG de los peces presenta variaciones intraespecíficas e interespecíficas, dependientes de factores como temperatura, salinidad, zona de captura, estación del año, alimento, tamaño, edad y etapa del ciclo de vida (Özogul et al., 2009). Los resultados del presente estudio concuerdan con lo anterior, ya que por ejemplo, las diferencias encontradas entre los AGS de la bacoreta y el pez vela pueden estar influidas por factores como su clima (la bacoreta es una especie tropical y el pez vela es subtropical).

Ácidos grasos monoinsaturados (AGM): Saito et al. (1999); Özogul et al. (2009) identificaron al C15:1, C16:1n-9, C17:1, C18:1n-9, C20:1n-9 y C22:1n-9, mientras que el C18:1n9t (elaídico) sólo se identificó en el presente trabajo. En este estudio no se identificaron algunos ácidos grasos monoinsaturados (AGM) los cuales fueron detectados en especies del Mar Mediterráneo, del Pacífico canadiense y del Mar del este de China (Huynh et al., 2007; Özogul et al., 2009; Saito et al., 1999), lo cual confirma que los AG están influenciados por factores como la ubicación geográfica y sus características asociadas.

El AGM más abundante identificado en todas las especies analizadas fue el C18:1n-9; lo mismo ocurrió en los estudios de Huynh et al. (2007), Njinkoué et al. (2002), Romero et al. (2000) y de Tanakol et al. (1999). Özogul et al. (2009) encontraron al C16:1 como el AGM más abundante en cuatro de 34 especies, con valores desde 13.31 hasta 19.61\%AGT. En el presente estudio, el segundo AGM más abundante excepto en la bacoreta y la mojarra rayada, fue el C16:1n-9.

Ciertos peces marinos son capaces de incorporar grandes cantidades de ácido elaídico y oleico (C18:1n-9t y C18:1n-9, respectivamente) en sus lípidos corporales, principalmente aquellas especies de climas cálidos (Halver, 1980). En las diez especies analizadas, el ácido elaídico se presentó en un intervalo muy amplio, desde una concentración traza de 0.01 hasta $32.40 \mathrm{mg} / 100 \mathrm{~g}$. El contenido de ácido oleico en las especies analizadas fue desde
24.00 en el pez vela, hasta $278.50 \mathrm{mg} / 100 \mathrm{~g}$ en la tilapia del Mozambique. La literatura reporta que aquellas especies que incluyen en su alimentación importantes cantidades de ácido linoleico (C18:2n-6) o alfa-linolénico (C18:3n-3) presentan menor concentración de los ácidos C18:1n-9t y C18:1n-9 en sus tejidos (Halver, 1980). Es probable que las variaciones de dichos ácidos en las especies analizadas se deban a múltiples factores, entre ellos la alimentación de los peces, elemento determinante para su composición (Steffens, 1997).

Los AGM son uno de los componentes más importantes del filete de algunos pescados, aunque su contribución a la composición de otros órganos (como hígado, intestino o hueva) es menor. Njinkoué et al. (2002) encontraron que tanto los AGS como los AGM son generalmente abundantes en especies de temperaturas templadas o calurosas; mientras que se observa lo contrario en especies de regiones de baja temperatura.

\section{Ácidos grasos poliinsaturados (AGP):} Entre 6 y 10 de los ácidos grasos poliinsaturados (AGP) identificados en el presente estudio también fueron identificados por otros autores (Huynh et al., 2007; Njinkoué et al., 2002; Özogul et al., 2009; Passi et al., 2002; Romero et al., 2000; Saito et al., 1999; Tanakol et al., 1999). El AGtrans C18:2n-6t (linolelaídico) sólo se identificó en el presente trabajo. El AGP más abundante en las especies estudiadas fue el DHA, excepto en la tilapia del Mozambique, probablemente por su origen. En 88 especies de pescado analizadas por otros autores, 67 presentaron al DHA como el AGP más abundante, mientras que en 21 se cuantificó con la mayor concentración al EPA (Huynh et al., 2007; Njinkoué et al., 2002; Özogul et al., 2009; Passi et al., 2002; Romero et al., 2000; Saito et al., 1999; Tanakol et al., 1999).

En un estudio realizado sobre especies comestibles de la Isla de Pascua (Romero et al., 2000) se encontró que las magras presentaron un contenido de C20:4n-6 (ácido araquidónico) desde 4.8 hasta $98.8 \mathrm{mg} / 100 \mathrm{~g}$. Los valores del presente estudio, salvo el reportado para 
el ronco amarillo, corresponden al intervalo presentado por este autor. Los ácidos araquidónico, EPA y DHA fueron los que se encontraron en mayor cantidad en todas las especies, lo que refleja su esencialidad para los peces marinos (Bell, Henderson \& Sargen, 1986).

Los AGP de los peces son específicos para cada especie y se relacionan con factores ambientales, dietarios, estacionales y del ciclo de vida del pez (Passi et al., 2002). Los resultados del presente trabajo lo confirman, ya que se encontraron diferencias significativas y numéricas en las especies analizadas según los diferentes factores bióticos y abióticos.

En los resultados reportados por otros autores se identificó el siguiente orden en la concentración de $\mathrm{AG}$ en un total de 89 especies de pescados marinos: AGS $>$ AGP $>$ AGM en 20 especies (sp.), AGS $>$ AGM $>$ AGP 24 sp., AGP $>$ AGS $>$ AGM 24 sp., AGP $>$ AGM $>$ AGS 6 sp., AGM $>$ AGS $>$ AGP 13 sp. y AGM>AGP>AGS 2 sp. (Huynh et al., 2007; Njinkoué et al., 2002; Özögul et al., 2009; Passi et al., 2002; Romero et al., 2000; Saito et al., 1999; Tanakol et al., 1999; Usydus et al., 2012).

AG n-3 y n-6: Con respecto a los AGn-3 y $\mathrm{n}-6$, en otros estudios se ha reportado un intervalo desde 12.66 hasta 57.1\%AGT (Huynh et al., 2007; Özogul et al., 2009; Passi et al., 2002; Saito et al., 1999; Tanakol et al., 1999). La concentración de AGn-3 del presente estudio es similar al rango anterior.

Brunner, Jones, Friel \& Bartley (2009) indicaron que las especies marinas pelágicas se consideran buena fuente de AGn-3 para humanos, seguidas de algunas especies demersales. En el presente trabajo se identificaron dos especies demersales (el ronco amarillo y la lengua esbelta) con el mayor contenido de AGn-3, seguidas de dos especies pelágicas (la bacoreta y el tolete).

Los resultados del presente estudio son consistentes con lo reportado por Huang, Bülbül, Wen, Glew \& Ayaz (2012), y confirman la aseveración de que a menor temperatura ambiental, mayor contenido de AGP (Halver,
1980; Lewis, 1962). Romero et al. (2000) encontraron gran variedad en la concentración de EPA y DHA en siete especies de pescado de la costa chilena, con una concentración de EPA desde 6.9 hasta $142.8 \mathrm{mg} / 100 \mathrm{~g}$, y DHA de 5.0 a $191.5 \mathrm{mg} / 100 \mathrm{~g}$.

La concentración de AGn-6 en las especies analizadas coincide con estudios previos que reportan un porcentaje desde 1.69 hasta 25.5\%AGT (Huynh et al., 2007; Özogul et al., 2009; Passi et al., 2002; Saito et al., 1999; Tanakol et al., 1999).

Las especies marinas se caracterizan por bajos niveles de C18:2n-6 (ácido linoleico) y C18:3n-3 (ácido linolénico) y altas concentraciones de EPA y DHA. En el presente estudio, el ácido linoleico en \% AGT se encontró desde 1.02 hasta 4.88 (en el lairón y el corviñón ocelado, respectivamente), mientras que Özogul et al. (2009) reportaron concentraciones desde 0.06 hasta 3.48\% AGT; Passi et al. (2002), reportaron de 0.9 hasta $3.8 \%$ AGT; Romero et al. (2000) de 0.3 a $2.4 \%$ AGT y Tanakol et al. (1999) desde 1.1 hasta 2.8\% AGT. En las especies analizadas el ácido linolénico se encontró de 0.19 a $6.32 \%$ AGT (el ronco amarillo y la tilapia del Mozambique, respectivamente); por otro lado, Özogul et al. (2009) reportaron desde 0.04 hasta 3.68\% AGT, Passi et al. (2002), de 0.04 hasta $1.9 \%$ AGT; Romero et al. (2000) de 0.9 a $3.8 \%$ AGT y Tanakol et al. (1999) desde 0.52 a $1.8 \%$ AGT.

Según Usydus et al. (2012), diferentes especies se caracterizan por tener variedad en sus perfiles de EPA y DHA, lo cual depende de la estación del año y lugar de captura. Saito et al. (1999), sugieren que el contenido de DHA de especies tropicales y subtropicales son menores a las concentraciones en especies de zonas árticas y subárticos. Ambas hipótesis se aplican a lo encontrado en el presente trabajo, ya que por ejemplo, el contenido de AGn-3 del salmón de Canadá (1000 a 1400mg/100g de porción comestible) es muy superior al valor de éstos ácidos grasos en todas las especies del presente estudio (Kris-Etherton et al., 2000).

La relación n6/n3 en los peces es muy importante para su estado de salud, pues se 
ha relacionado con algunas alteraciones metabólicas. El resultado del presente es similar a lo reportado por Özogul et al. (2009) de 0.04 a 0.91 . El promedio de la relación $\mathrm{n} 6 / \mathrm{n} 3$ en especies de pescado marinas es de 0.16 , la cual depende de la alimentación y se reduce conforme disminuye la temperatura del medio donde habitan. Cuando los lípidos de la alimentación de los peces de agua dulce son ricos en n-6, las especies son capaces de alterar esta relación en sus tejidos para aumentar el contenido de n-3, lo cual no ocurre en el ser humano (Halver, 1980). Cuando la relación n6/n3 es muy alta en la alimentación de los peces, se pueden llegar a desarrollar algunas patologías como cardiomiopatías, aumento en la incidencia de lesiones ateroescleróticas, disminución de la habilidad del hígado para desintoxicar xenobióticos y disminución de la resistencia a infecciones bacterianas (Sargent et al., 1993).

Los resultados del presente trabajo, principalmente los referentes al EPA y DHA, tienen un gran potencial en investigaciones in vivo asociadas a patologías humanas. Algunos estudios han empleado aceites de pescado como estrategia no invasiva contra lesiones de isquemia y reperfusión en hígado de rata y para la reducción del estrés oxidativo en hígado de ratas (Mardones et al., 2012; Valenzuela et al., 2011), con la información del presente trabajo se podrían seleccionar las especies con mayor contenido de estos ácidos grasos para sustituir el empleo de aceites de pescado. En el caso de estudios clínicos, el empleo de los pescados aquí analizados proporcionaría no sólo el beneficio de los ácidos grasos sino también el de su proteína de alto valor biológico, sus vitaminas y minerales, así como su bajo contenido de colesterol.

En conclusión, los factores bióticos y abióticos juegan un papel muy importante en la variación de la composición de ácidos grasos en los pescados analizados. La zona de captura (origen) y el clima (tropical y subtropical) impactaron en mayor medida, ya que se encontró un mayor número de especies con diferencias significativas entre sí en estos factores. Factores bióticos también son determinantes en la variación del perfil de ácidos grasos. El clima tuvo un mayor impacto en la concentración de los AGP, principalmente para $\mathrm{EPA}+\mathrm{DHA}$, ya que las especies subtropicales presentaron una concentración $42.10 \%$ mayor que las tropicales.

Es importante tomar en cuenta que los valores presentados en este trabajo van a variar según los cambios que se presenten en los diferentes ecosistemas y características de cada especie. Este tipo de estudio sirve como base para llevar a cabo futuras investigaciones sobre el beneficio que el consumo de de pescado tiene en el manejo de diversas patologías, en las cuales es necesario calcular la ingestión de algunos nutrimentos contenidos en el pescado con propiedades antiinflamatorias, cardioprotectoras, antitrombóticas, etc. como son los ácidos grasos n-3. Estudios como el presente permiten que los profesionales de la salud tengan herramientas para ofrecer más y mejores alimentos a personas que padecen enfermedades no transmisibles (Castro, Miranda \& Montaño, 2009; Molina de Salazar, 2009).

\section{AGRADECIMIENTOS}

Los autores agradecen a Jorge Toral Peña por proveer las especies de pescado analizadas en el presente trabajo y a José Luis Castro Aguirre $\dagger$ por su ayuda en la identificación de algunas especies.

\section{RESUMEN}

Los factores bióticos y abióticos determinan la composición de ácidos grasos (AG) en los peces, información relevante por la asociación existente entre su consumo y los beneficios para la salud humana. El objetivo de esta investigación fue identificar la variación en la concentración de AG, según diferentes factores, en diez especies marinas de pescado comestible en México: Euthynnus alletteratus, Sciaenops ocellatus, Bairdiella chrysoura, Sphyraena guachancho, Symphurus elongatus, Istiophorus platypterus, Ophichthus rex, Eugerres plumieri, Eucinostomus entomelas y Oreochromis mossambicus. La determinación de AG se llevó a cabo mediante cromatografía de gases. El contenido de lípidos totales varió entre 0.93 y $1.95 \mathrm{~g} / 100 \mathrm{~g}$ en E. entomelas y $O$. mossambicus, respectivamente. E. alletteratus, B. chrysoura, $S$ elongatus, I. platypterus, O. rex y 
E. plumieri presentaron el siguiente orden en la concentración de AG: AG poliinsaturados (AGP) $>$ AG saturados (AGS)>AG monoinsaturados (AGM). S. ocellatus, S. guachancho y E. entomelas: AGS $>$ AGP $>$ AGM; mientras que tan solo $O$. mossambicus presentó AGS $>$ AGM $>$ AGP. Este tuvo la mayor concentración de AGS $(559.40 \mathrm{mg} / 100 \mathrm{~g})$ y AGM $(422.60 \mathrm{mg} / 100 \mathrm{~g})$, mientras que $B$. chrysoura presentó el mayor contenido (mg/100g) de AGP (663.03), AGn-3 (514.03), EPA+DHA (506.10) y AGn-6 (145.80). Factores bióticos y abióticos influyeron significativamente en la concentración de AG. Las especies subtropicales presentaron una concentración $42.1 \%$ mayor de EPA+DHA que las tropicales. Los valores presentados pueden cambiar según modificaciones en el ecosistema y en las características de cada especie, sin embargo, el conocimiento aquí generado es útil para mejorar las recomendaciones sobre el consumo de pescado.

Palabras clave: ácidos grasos, pescados marinos, factores bióticos, factores abióticos, México.

\section{REFERENCIAS}

Association of Official Analytical Chemists. (1996). Methods of Analysis. Washington, D. C.: Autor.

Bell, M. V., Henderson, R. J., \& Sargent, J. R. (1986). The role of polyunsaturated fatty acids in fish. Comparative Biochemistry and Physiology, 83B, 711-719. Doi: 0305-0491/86.

Bligh, E. G., \& Dyer, W. J. (1959). A rapid method of total lipid extraction and purification. Canadian Journal of Biochemistry and Physiology, 37, 911-917.

Brookhyser, J. (2006). Omega 3 Fatty Acids. Journal of Renal Nutrition, 16, e7-e10.

Brunner, E. J., Jones, P. J. S., Friel, S., \& Bartley, M. (2009). Fish, human health and marine ecosystem health: policies in collision. International Journal of Epidemiology, 38, 93-100. Doi: 10.1093/ije/dyn157|.

Castro-González, M. I., Ojeda, V. A., Montaño, B. S., Ledesma, C. E., \& Pérez-Gil, R. F. (2007). Evaluación de los ácidos grasos n-3 de 18 especies de peces marinos mexicanos como alimentos funcionales. Archivos Latinoamericanos de Nutrición, 57, 85-93.

Castro-González, I., Miranda, D., \& Montaño, S. (2009). Evaluation of phosphorus, protein and n-3 fatty acid content in 15 marine fish species identifies the species most beneficial to renal patients. Journal of Renal Nutrition, 19, 462-468.

D’Armas, H., Yáñez, D., Reyes, D., \& Salazar, G. (2010). Composición de ácidos grasos de los caracoles marinos Phyllonotus pomum y Chicoreus brevifrons (Gastropoda: Muricidae). Revista de Biología Tropical, 58, 645-654.
Dey, I., Buda, C., Wiik, T., Halver, J. E., \& Farkas, T. (1993). Molecular and structural composition of phospholipid membranes in livers of marine and freshwater fish in relation to temperature. Proceeding of the National Academy of Sciences, 90, 7498-7502.

Food and Agriculture Organization. (2012). La lista ASFIS de especies para los fines de estadísticas de pesca. Recuperado de http://www.fao.org/fishery/collection/ asfis/es.

Folch, J., Lees, M., \& Sloane Stanley, G. H. (1957). A simple method for the isolation and purification of total lipids from animal tissues. The Journal of Biological Chemistry, 226, 497-509.

Froese, R., \& Pauly, D. (2011). FishBase. Recuperado de www.fishbase.org.

Ghaddar, S., \& Saoud, I. P. (2012). Seasonal Changes in Phosphorus Content of Fish Tissue as They Relate to Diets of Renal Patients. Journal of Renal Nutrition, 22, 67-71.

Gidding, S. S., Lichtenstein, A. H., Faith, M. S., Mennella, J. A., Popkin, B., Rowe, J., Van Horn, L., \& Whitsel, L. (2009). Implementing American Heart Association Pediatric and Adult Nutrition Guidelines: A Scientific Statement From the American Heart Association Nutrition Committee of the Council on Nutrition, Physical Activity and Metabolism, Council on Cardiovascular Disease in the Young, Council on Arteriosclerosis, Thrombosis and Vascular Biology, Council on Cardiovascular Nursing, Council on Epidemiology and Prevention, and Council for High Blood Pressure Research. Circulation, 119, 1161-1175.

Halver, J. E. (1980). Fish Feed Technology. RomaFAO/ UNDP: Aquaculture Development and Coordination Programme.

Hedelin, M., Löf, M., Olson, M., Lewander, T., Nilsson, B., Hultman, C. M., \& Weiderpass, E. (2010). Dietary intake of fish, omega-3, omega-6 polyunsaturated fatty acids and vitamin D and the prevalence of psychotic-like symptoms in a cohort of 33000 women from the general population. BMC Psychiatry, 10, 38 .

Huang, C., Bülbül, U., Wen, P. C., Glew, R. H., \& Ayaz, F. A. (2012). Fatty Acid Composition of 12 Fish Species from the Black Sea. Journal of Food Science, 13, 3077-3082.

Huynh, M. D., Kitts, D. D., Hu, C., \& Trites, A. W. (2007). Comparison of fatty acid profiles of spawning and non-spawning Pacific herring, Clupea harengus pallasi. Comparative Biochemistry and Physiology Part B, 146, 504-511. Doi: 10.1016/j. cbpb.2006.11.023.

Kris-Etherton, P. M., Taylor, D. S., Yu-Poth, S., Hutg, P., Moriarty, K., Fishell, V., Hargrove, R. L., Zhao, G., \& Etherton, T. D. (2000). Polyunsaturated fatty acids 
in the food chain in the United States. The American Journal of Clinical Nutrition, 71, 179S-188S.

Kris-Etherton, P. M., Harris, W. S., Lawrence, J., \& Nutrition Committee. (2002). Fish Consumption, Fish Oil, Omega-3 Fatty Acids, and Cardiovascular Disease. Circulation, 106, 2747-2757.

Lewis, R. W. (1962). Temperature and pressure effects on the fatty acids of some marine ectotherms. Comparative Biochemistry and Physsiology, 6, 75-89.

Lichtenstein, A. H., Appel, L. J., Brands, M., Carnethon, M., Daniels, S., Franch, H. A., Franklin, B., KrisEtherton, P., Harris, W. S., Howard, B., Karanja, N., Lefevre, M., Rudel, L., Sacks, L., Van Horn, L., Winston, M., \& Wylie-Rosett, J. (2006). Diet and Lifestyle Recommendations Revision 2006: A Scientific Statement from the American Heart Association Nutrition Committee. Circulation, 114, 82-96.

Mardones, M., Valenzuela, R., Romanque, P., Covarrubias, N., Anghileri, F., Fernández, V., Videla, L. A., \& Tapia, G. (2012). Prevention of liver ischemia reperfusion injury by a combined thyroid hormone and fish oil protocol. The Journal of Nutritional Biochemistry, $23,1113-1120$

Molina de Salazar, D. I. (2009). Evidencia clínica de los ácidos grasos omega-3 procedentes de los peces en prevención primaria y secundaria. Revista Colombiana de Cardiología, 16, 9-11.

Morrison, W. R., \& Smith, L. M. (1964). Praparation of fatty acids methyl esters and dimethylacetals from lipids with boron fluoride- methanol. The Journal of Lipid Research, 5, 600-608.

Njinkoué, J. M., Barnathan, G., Miralles, J., Gaydou, E. M., \& Samb, A. (2002). Lipids and fatty acids in muscle, liver and skin of three edible fish from the Senegalese coast: Sardinella maderensis, Sardinella aurita and Cephalopholis taeniops. Comparative Biochemistry and Physiology Part B, 131, 395-402. Doi: 1096-4959/02.

Nurnadia, A. A., Azrina, A., \& Amin, I. (2011). Proximate composition and energetic value of selected marine fish and shellfish from the West coast of Peninsular Malasya. International Food Research Journal, 18, 137-148.

Ortiz, H., \& Bosch, V. (1994). Ácidos grasos en pescados de mar y de río de consumo frecuente en Venezuela. Anales Venezolanos de Nutrición, 7, 27-30.

Özogul, Y., Özogul, F., Cícek, E., Polat, A., \& Kuley, E. (2009). Fat content and fatty acid compositions of 34 marine water fish species from the Mediterranean Sea.
International Journal of Food Sciences and Nutrition, 60, 464-475. Doi: 10.1080/09637480701838175.

Passi, S., Cataudella, S., Di Marco, P., De Simone, F., \& Rastrelli, L. (2002). Fatty Acid Composition and Antioxidant Levels in Muscle Tissue of Different Mediterranean Marine Species of Fish and Shellfish. Journal of Agricultural and Food Chemistry, 50, 7314-7322. Doi: 10.1021/jf020451y.

Patton, J. S. (1975). The effect of pressure and temperature on phospholipid and triglyceride fatty acids of fish white muscle: A comparison of deepwater and surface marine species. Comparative Biochemistry and Physiology, 52, 105-110.

Perea, A., Gómez, E., Mayorga, Y., \& Triana, C. Y. (2008). Caracterización nutricional de peces de producción y consumo regional en Bucaramanga, Colombia. Archivos Latinoamericanos de Nutrición, 58, 91-97.

Pérez, C. (2000) Técnicas de muestreo estadístico. Teoría, práctica y aplicaciones informáticas. España: Alfaomega-Rama.

Rasoarahona, J. R. E., Barnathan, G., Bianchini, J. P., \& Gaydou, E. M. (2005). Influence of season on the lipid content and fatty acid profiles of three tilapia species (Oreochromis niloticus, O. macrochir and Tilapia rendalli) from Madagascar. Food Chemistry, 91, 683-694. Doi: 10.1016/j.foodchem.2004.07.001.

Romero, N., Robert, P., Masson, L., \& Pineda, R. (2000). Composición en ácidos grasos y proximal de siete especies de pescado de Isla de Pascua. Archivos Latinoamericanos de Nutrición, 50, 304-308.

Saito, H., Yamashiro, R., Alasalvar, C., \& Konno, T. (1999). Influence of Diet on Fatty Acids of Three Subtropical Fish, Subfamily Caesioninae (Caesio diagramma and C. tile) and Family Siganidae (Siganus canaliculatus). Lipids, 34, 1973-1082.

Sánchez-Villegas, A., Henríquez, P., Figueiras, A., Ortuño, F., Lahortiga, F., \& Martínez-González, M. A. (2007). Long chain omega-3 fatty acids intake, fish consumption and mental disorders in the SUN cohort study. European Journal of Nutrition, 46, 337-346.

Sargent, J. R., Bell, J. G., Bell, M. V., Henderson, R. J., \& Tocher, D. R. (1993). Aquaculture: Fundamental and Applied Research. EUA: American Geophysical Union.

Sargent, J., Bell, G., McEvoy, L., Tocher, D., \& Estevez, A. (1999). Recent developments in the essential fatty acid nutrition of fish. Aquaculture, 177, 191-199. Doi: 0044-8486/99. 
StataCorp. (2007). Stata Statistical Software: Release 10. College Station, TX: StataCorp LP. Programa computacional.

Steffens, W. (1997). Effects of variation in essential fatty acids in fish feeds on nutritive value of freshwater fish for humans. Aquaculture, 151, 97-119. Doi: 0044-8486/97.

Systat Software. SigmaPlot 11.0. [CD-ROM] San Jose, California, USA. Programa computacional.

Tanakol, R., Yazici, Z., Sener, E., \& Sencer, E. (1999). Fatty Acid Composition of 19 Species of Fish from the Black Sea and the Marmara Sea. Lipids, 34, 291-297.

Tang, H., Chen, L., Xiao, C., \& Wu, T. (2009). Fatty acid profiles of muscle from large yellow croaker (Pseudosciaena crocea $R$.) of different age. Journal of Zhejiang University - Science B, 10, 154-158.

Usydus, Z., Szlifder-Richert, J., \& Adamcdzyk, M. (2012). Variations in proximate composition and fatty acid profiles of Baltic sprat (Sprattus sprattus balticus). Food Chemistry, 130, 97-103. Doi: 10.1016/j. foodchem.2011.07.003.

University of Maryland Medical Center. (2011). Omega 3. Recuperado de http://www.umm.edu/altmed/articles/omega-3-000316.htm.

Valenzuela, R., \& Videla, L. A. (2011). The importance of the long-chain polyunsaturated fatty acid n-6/n-3 ratio in development of non-alcoholic fatty liver associated with obesity. Food \& Function, 2, 644-648.

Vrablík, M., Prusíková, M., Snejdrlová, M., \& Zlatohlávek, L. (2009). Omega 3 fatty acids and cardiovascular disease risk: do we understand the relationship? Physiological Research, 58, S19-S26.

Wall, R., Ross, R. P., Fitzgerald, G. F., \& Stanton, C. (2010). Fatty acids from fish: the anti-inflammatory potential of long-chain omega-3 fatty acids. Nutrition Reviews, 69, 280-289. Doi: 10.1111/j.1753-4887.2010.00287.x. 